Article

\title{
Multilayered Artificial Dura-Mater Models for a Minimally Invasive Brain Surgery Simulator
}

\author{
Masaru Takeuchi ${ }^{1, *} \mathbb{0}$, Shusaku Hayakawa ${ }^{2}$, Akihiko Ichikawa ${ }^{2}$, Akiyuki Hasegawa ${ }^{2}$, \\ Yasuhisa Hasegawa ${ }^{1}$ and Toshio Fukuda ${ }^{2}$ \\ 1 Department of Micro-Nano Mechanical Science and Engineering, Nagoya University, \\ Nagoya 4648603, Japan; yasuhisa.hasegawa@mae.nagoya-u.ac.jp \\ 2 Department of Mechatronics Engineering, Meijo University, Nagoya 4688502, Japan; \\ 183432016@ccalumni.meijo-u.ac.jp (S.H.); ichikawa@meijo-u.ac.jp (A.I.); haak.abmes@gmail.com (A.H.); \\ tofukuda@meijo-u.ac.jp (T.F.) \\ * Correspondence: takeuchi@mein.nagoya-u.ac.jp; Tel.: +81-52-789-2717
}

Received: 6 November 2020; Accepted: 11 December 2020; Published: 16 December 2020

\section{Featured Application: Surgical Simulator; Artificial Human Model.}

\begin{abstract}
In this study, new artificial dura-mater models were developed using a multilayered structure of a rubber material (represent an elastic component of a dura-mater) and a fiber sheet (represent fiber component of a dura-mater). The artificial dura-mater models were prepared for use in a brain surgery simulator, especially for transnasal pituitary surgery. The mechanical characteristics of the artificial dura-mater models were tested to check the similarities with porcine dura-mater. Tensile stress, viscoelasticity, and the cutting force generated by microscissors were tested to evaluate the fabricated models. Neurosurgeons also assessed the dura-mater models to evaluate their characteristics. The results indicate that these models made of two different non-woven fiber sheets emulated accurately the actual dura-mater.
\end{abstract}

Keywords: artificial dura-mater; surgical simulator; layered structure; brain surgery

\section{Introduction}

Minimally invasive surgeries have advanced recently. This has led to the necessity of enhanced surgical skills. To achieve this, a surgical simulator has been used because it does not require experimental animals or actual patients for training. Typically, two types of surgical simulators have been developed: one is a virtual reality (VR) simulator based on VR technology, and the other is a physical (real-type) simulator that uses an artificial organ model. The VR simulators can construct and display surgical targets and environments flexibly, such as body parts (skin, bones, organs, blood vessels), and surgical tools in virtual spaces. In this way, physicians demonstrate and improve their surgical skills through constructed VR environments [1-4]. The VR simulators easily generate various cases of diseases or injuries, and surgical tools are easily changed in the VR environment. Hence, physicians are trained in arbitrary conditions by a VR simulator to improve their surgical skills or to practice the testing of new surgical instruments. However, the VR simulators have some limitations because they do not allow to use of actual surgical instruments or devices during a surgical simulation. Generally, the handling parts of VR simulators are fixed by the haptic device, and they are not easily exchanged to other handles to emulate actual surgical tools.

A physical (real-type) surgical simulator allows to use of practical surgical tools in simulations. Models of surgical parts (organs, blood vessels, skin, etc.) are created to achieve similar properties of human organs, and the surgical training is conducted using the same environments of practical 
surgeries [5-8]. Different disease or injury cases are reconstructed and simulated by replacing organ models or body parts. However, mechanical characteristics, such as stiffness, fragility, and cutting forces in actual body parts and artificial models, still have some differences. For example, Joseph F.J. et al. developed a physical simulator able to reproduce the experience of clipping an intracranial aneurysm using a 3D printed model of the skull, brain, and arteries [9]. They found that a large majority of neurosurgeons answered the developed physical simulator was better than conventional virtual reality-based training methods.

In this study, dura-mater models were developed for a simulator of minimally invasive brain surgery. The dura-mater models were fabricated by using both elastic and fiber materials to emulate the actual dura-mater in surgical procedures, such as sectioning operations by surgical microscissors. The mechanical properties of the dura-mater models were tested and compared with those of the porcine dura-mater to evaluate them quantitatively. Sensory tests of fabricated dura-mater models were finally conducted by neurosurgeons to ascertain whether the dura-mater models have the necessary properties to allow their use in a surgical simulator.

\section{Dura-Mater}

Figure 1 shows a sagittal view of a human head. The bony and membranous parts are layered from the skin to the brain matter. The dura-mater is one of the three membrane layers of the brain (pia mater, arachnoid, and dura-mater) that cover the central nervous system on the outermost cover. It is divided into two different layers. One is the spinal dura-mater and the other is the cranial dura-mater. The spinal dura-mater covers the spinal nerve, and the cranial dura-mater is attached to the inner surface of the skull and covers the entire brain. Several studies quantified the mechanical characteristics of dura-mater. McGarvey et al. obtained a tensile strength of a human spinal dura-mater at $61.5 \pm 9.6 \mathrm{MPa}$ in the linear region with elongation strain in the range of $15-20 \%$ in a uniaxial tensile test [10]. Shetye et al. showed a tensile strength value of $5.6 \mathrm{MPa}$ in the axial direction and 1.6 MPa in the circumferential direction using a biaxial tensile test of a sheep's spinal dura-mater [11]. Kizmazoglu et al. reported that the cranial human dura-mater had a mean tensile strength of $\sim 7 \mathrm{MPa}$ [12]. Hence, the human dura-mater has a tensile strength of the order of several MPa. However, conventional studies just focused on the tensile test to measure Young's modulus of the dura-mater, and the mechanical properties of the artificial dura-mater related to surgical operations, such as the cutting force by microscissors, have not been quantified.

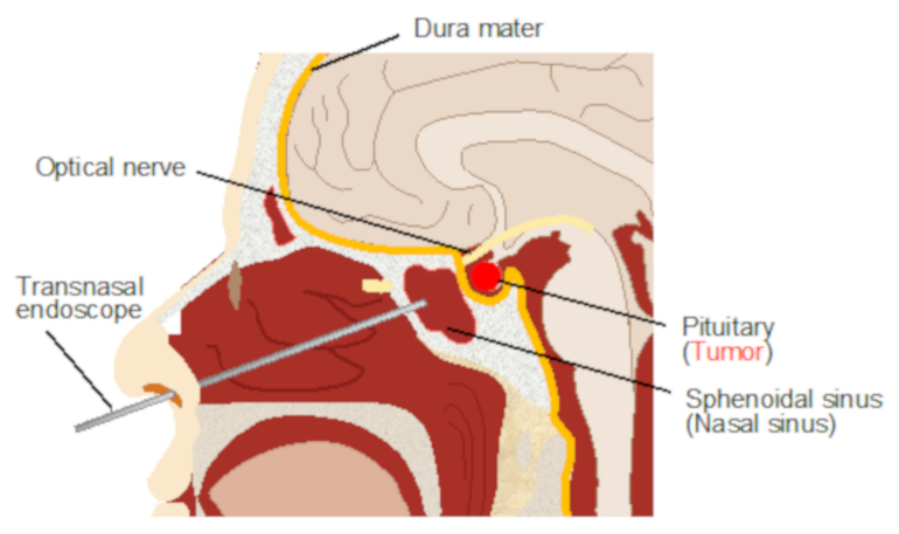

Figure 1. Sagittal view of the brain and its structures with a transnasal endoscope for neurosurgery.

Existing artificial dura-maters which were clinically used, such as Gore-Tex ${ }^{\circledR}$ Expanded Cardiovascular Patch (W.L. Gore \& Associates Inc., Flagstaff, AZ, USA), Durepair ${ }^{\circledR}$ (Medtronic Inc., Goleta, CA, USA), and Tutopatch ${ }^{\circledR}$ (Tutogen Medical GmbH, Neunkirchen am Brand, Germany), were generally used for closing holes on a skull after surgery [12]. The Gore-Tex ${ }^{\circledR}$ Expanded Cardiovascular Patch is made of expanded polytetrafluoroethylene (ePTFE), Durepair ${ }^{\circledR}$ is a porosity 
sheet made of collagen type II and III. Tutopatch ${ }^{\circledR}$ is also a collagenous membrane. Those artificial dura-maters have been developed to be used in vivo (for actual brain surgeries). Therefore, those models were not considered to be used in vitro (dry condition) and the mechanical properties of the artificial dura-mater related to surgical operations, such as the cutting force generated by microscissors, have not been considered. The collagenous membranes are not suitable for surgical simulators because their mechanical properties depend on wet or dry conditions. Some conventional real type surgical simulators did not have a dura-mater model because their target surgery did not have an important role in cutting or suturing the dura-mater [13-15]. Several studies developed artificial dura-mater models for surgical simulators of brain surgeries. For example, Craven C. et al. developed an anatomical replica for training young neurosurgeons [16]. A latex dura-mater model was used to mimic the actual dura-mater. However, the model made of latex could not represent the elastic fibers in dura-mater, such as collagen and elastin fibers. Eastwood K. W. et al. developed a dura-mater model by laminating silicone-okawara papers. The paper fibers could mimic the elastic fibers in dura-mater, but the mechanical properties of the developed dura-mater model were not evaluated [17]. Hence, new dura-mater models for the surgical simulators of brain surgery are desired.

This paper shows the development of dura-mater models for a brain surgical simulator, especially for transnasal pituitary surgery. This type of surgery is known as minimally invasive surgery, and pituitary gland tumors are excised by surgical instruments following their insertion in the nose. Figure 1 indicates a sagittal view of the brain and its structures with a transnasal endoscope during the transnasal pituitary surgery. The surgical procedures should be held in a limited space of the human head. The surgical area contains important anatomical structures, including the carotid artery and optic nerve. The dura-mater should be severed to allow access to pituitary gland tumors during the surgery, and a hole on the dura-mater has to be closed by suturing to prevent leakage of spinal fluid. Suturing the dura-mater in the narrow space needs to enhanced surgical techniques $[18,19]$.

New models of artificial dura-mater were developed by the combination of a rubber material (acrylic rubber) and a fiber sheet (non-woven polypropylene fabrics) to mimic the elastic components and fiber components in a dura-mater. The mechanical characteristics of the dura-mater models related to the surgical operation were quantified, and the results were compared with those from a porcine dura-mater. The dura-mater models were used for sensory tests by neurosurgeons to ascertain whether the developed dura-mater models have adequate properties to be installed as a surgical simulator of brain surgery.

\section{Fabrication Procedure of Dura-Mater Models}

The dura-mater is made of a combination of biological cells and intercellular materials, for example, collagen, elastin, and an interstitial material indicated in Figure 2a,b [20-22]. Generally, the dura-mater contains approximately $70 \%$ water, $20 \%$ collagen, $10 \%$ elastin, and a small number of other molecules. The tissue exhibits nonlinearity both in terms of the material and geometry. It has various characters, such as incompressibility, inhomogeneity, viscoelasticity, and anisotropy. A new dura-mater model should be better to have similar mechanical properties for use as a surgical simulator model.
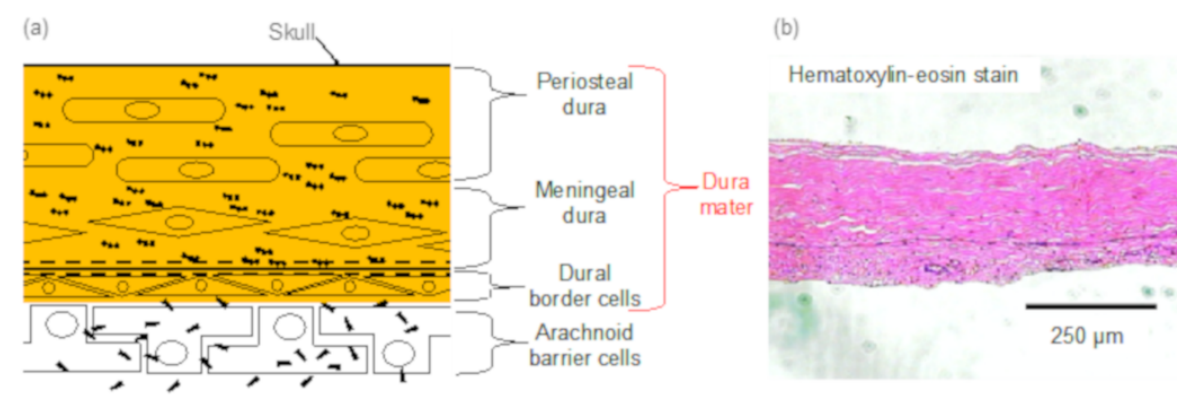

Figure 2. Dura-mater: (a) Cross-sectional structure of dura-mater; (b) Cross-sectional photograph of porcine dura-mater after hematoxylin-eosin (HE) staining. 
To emulate elastic fibers presented in an actual dura-mater, such as collagen and elastin fibers, non-woven polypropylene fabrics were employed to develop the dura-mater models in our previous paper [23]. In this study, two different non-woven fabrics were chosen for new dura-mater models, as indicated in Table 1. The dura-mater has collagen fibers, which have an anisotropic structure with different sizes of bundles [24]. To mimic the structure of collagen fibers in dura-mater, two different PP non-woven fabrics were employed. An acrylic rubber (BE-1, Yutaka Make, Osaka, Japan) was used for the elastic part of the dura-mater model. The acrylic rubber is an aqueous rubber, which contains $40 \%$ to $50 \%$ acrylic resin, $40 \%$ to $50 \%$ water, $3 \%$ defoamer, and $5 \%$ surfactant. The fabrication procedure of a dura-mater model is shown in Figure $3[23,25]$. At first, the non-woven fabric was cut to a size of $100 \times 100 \mathrm{~mm}^{2}$. The acrylic rubber was diluted with pure water at a weight ratio of 10:7. Two or three non-woven fabrics were stacked in a layer-by-layer manner, and the acrylic rubber aqueous solution was dip-coated. The coated acrylic rubber was dried at about $25^{\circ} \mathrm{C}$. The acrylic rubber aqueous solution was then spin-coated on one side of the non-woven fabrics. After drying the coated acrylic rubber, the aqueous solution of the acrylic rubber was spin-coated on the other side. In this study, five dura-mater models were prepared as a combination of two different fabrics and acrylic rubber (Figure 4). The dura-mater models shown in Figure 4a-c had two sheets of non-woven fabrics, and the dura-mater models shown in Figure 4d,e had three sheets of the non-woven fabrics. The surface of all dura-mater models was coated by the acrylic rubber. All dura-mater models were prepared at the same thickness that ranged between $0.02 \mathrm{~mm}$ and $0.03 \mathrm{~mm}$. The thickness was determined to be the same range of porcine dura-mater thickness to compare the mechanical properties of dura-mater models with the porcine dura-mater in the same condition. The human dura-mater has about twice a larger thickness than the porcine dura-mater [24]. The thickness of dura-mater models can be controlled by changing the thickness of the acrylic rubber layer, or a number of layers of non-woven fabrics. Figure $5 \mathrm{a}$ shows the fabricated dura-mater model made of acrylic rubber and non-woven fabrics (soft and soft).

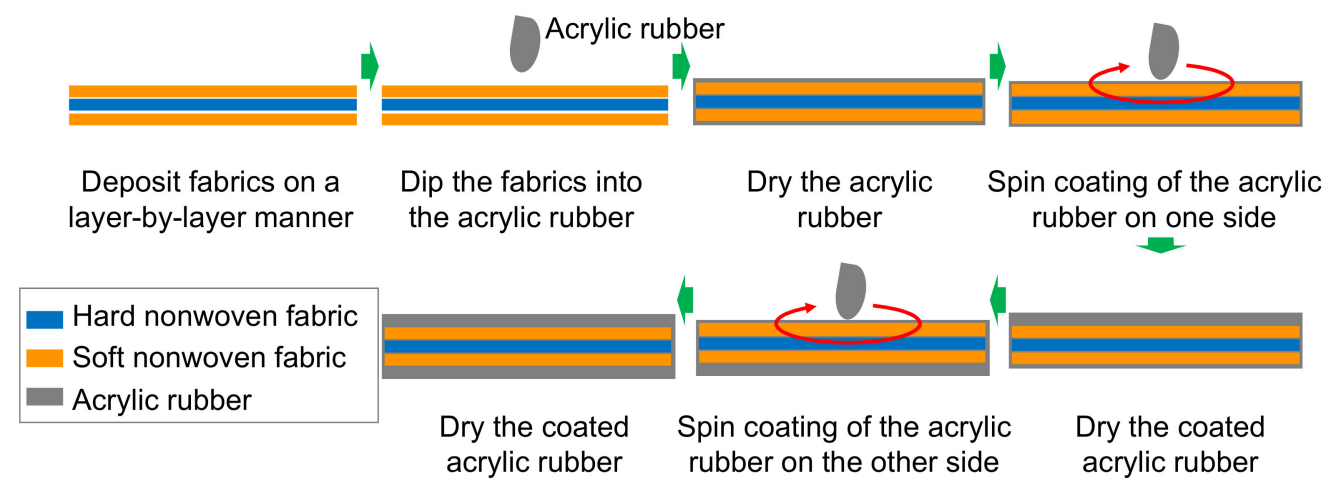

Figure 3. Schematic of the fabrication procedure of dura-mater models.

(a)

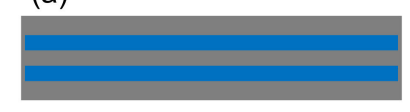

(d)

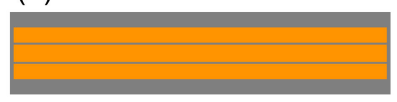

(b)

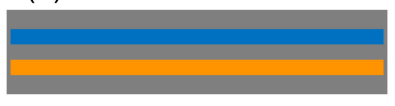

(e)

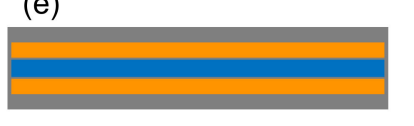

(c)

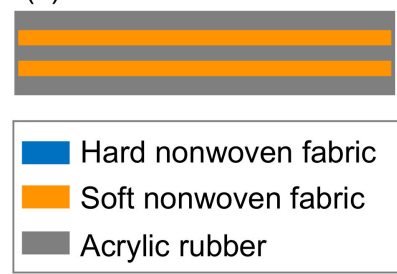

Figure 4. A cross-sectional structure of developed dura-mater models: (a) Acrylic rubber and non-woven fabric (hard and hard); (b) Acrylic rubber and non-woven fabric (hard and soft); (c) Acrylic rubber and non-woven fabric (soft and soft); (d) Acrylic rubber and non-woven fabric (soft and soft and soft); (e) Acrylic rubber and non-woven fabric (soft and hard and soft). 
(a)

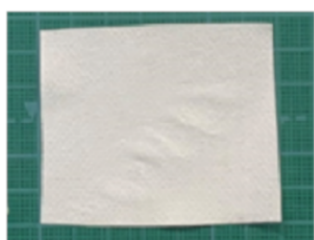

(b)

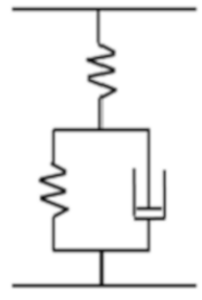

(c)

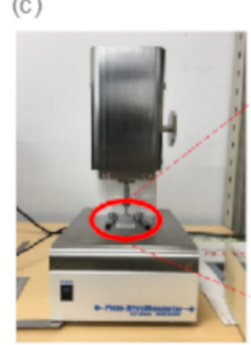

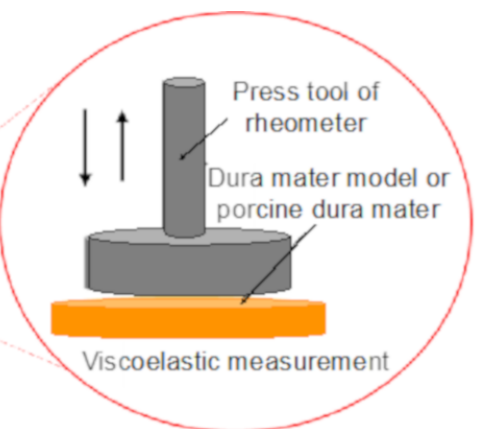

Figure 5. Viscoelastic measurements: (a) Fabricated dura-mater model; (b) Three-element viscoelastic model; (c) Measurement setup for viscoelasticity of dura-mater models.

Table 1. Fabric materials for dura-mater models.

Hard non-woven fabric
Thickness:
$120 \pm 10 \mu$
mFiber diameter:
$40 \pm 10 \mu \mathrm{m}$
$\begin{gathered}\text { Thickness: } \\ 80 \pm 10 \mu \\ \text { Soft non-woven fabric } \\ \text { 20 } 10 \mu \mathrm{m}\end{gathered}$

\section{Evaluation of Dura-Mater Models}

The evaluation of developed dura-mater models was conducted by measuring their mechanical characteristics and comparing them with that of porcine dura-mater. The porcine dura-mater was taken from the slaughtered pigs (6-7 months of age), which were purchased from the meat market in Nagoya, Japan. The porcine dura-mater was measured within $24 \mathrm{~h}$ after slaughter. Three measurements were tested to evaluate the developed dura-mater models: viscoelastic measurement, tensile test, and cutting test using surgical microscissors. The viscoelastic measurement was demonstrated by a rheometer, and the other two experiments were tested using a tensile tester with microscissors.

\subsection{Viscoelastic Measurements}

Dura-mater is known as a composite material that has viscoelastic properties [26,27]. Its viscoelasticity was measured to check if the developed dura-mater models show similar properties compared with those of the actual dura-mater. In this study, we used a three-element viscoelastic model to express the mechanical properties of dura-mater models. The three-element viscoelastic model has one spring and a Voigt model (Figure 5b). The three-element viscoelastic model exhibits a solid-like behavior in the condition of constant stress and a stress relaxation phenomenon over time in the condition of constant strain.

A rheometer employed with the three-element viscoelastic model was employed to quantify the viscoelasticity of dura-mater models. Storage and loss moduli were obtained from the measurements 
by a rheometer (PZ-Rheo NDS-1000, Syscom, Tokyo, Japan) with a probe that had $10 \mathrm{~mm}$ in diameter (Figure 5c). The storage modulus $G^{\prime}$ and loss modulus $G^{\prime \prime}$ were measured by the rheometer based on the following Equations (1) to (4).

$$
\begin{gathered}
G^{\prime}=\left(\frac{\tau_{\max }}{\gamma_{\max }}\right) \cos \delta \\
G^{\prime \prime}=\left(\frac{\tau_{\max }}{\gamma_{\max }}\right) \sin \delta \\
\gamma(t)=\frac{u(t)}{d} \\
\tau(t)=\frac{F(t)}{A}
\end{gathered}
$$

where $\delta$ represents the phase difference, $\gamma$ represents the shear strain, $u$ shows the displacement, $d$ indicates the sample thickness, $\tau$ indicates the applied stress, $F$ indicates the reactive force, and $A$ represents the sample area. Strain with a frequency of $3 \mathrm{~Hz}$ and an amplitude of $5 \%$ was applied to the samples, and the storage modulus $G^{\prime}$ and loss modulus $G^{\prime \prime}$ were calculated from Equations (1)-(3). Measurement of six different samples were carried out: porcine dura-mater and five different dura-mater models (Figure 4a-e). The measurement was repeated five times in each sample, and the highest and lowest values were eliminated from the results.

Figure 6 indicates the storage modulus $G^{\prime}$ and loss modulus $G^{\prime \prime}$ measured in each sample. The dura-mater model in Figure 4a yields relatively similar $E^{\prime}$ and $E^{\prime \prime}$ values, while the dura-mater model in Figure $4 b, c$ had similar properties with those of the porcine dura-mater from the viewpoint of $G^{\prime}$. The results indicate that the dura-mater models with two-layered fabrics yielded better results compared with the three-layered fabrics from the viewpoint of viscoelasticity. The differences in the results may be attributed to the differences in thickness in the acrylic rubber.
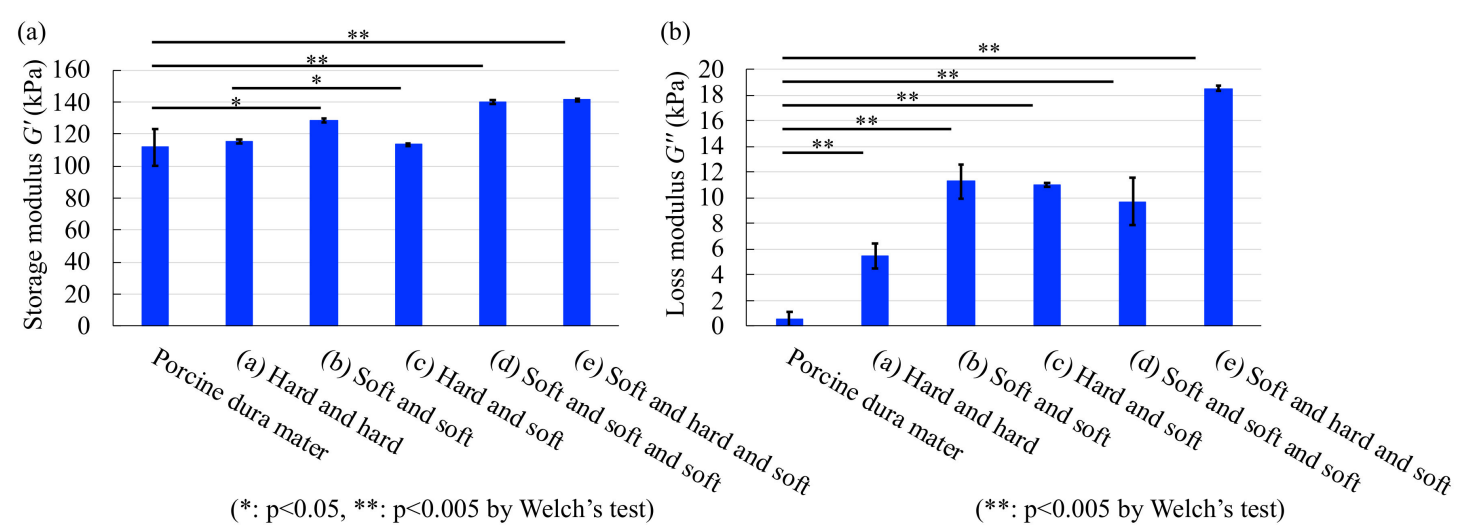

Figure 6. Measured viscoelastic results: (a) Storage modulus of porcine dura-mater and each dura-mater model; (b) Loss modulus of porcine dura-mater and each dura-mater model.

\subsection{Tensile Test}

Mechanical characteristics of developed dura-mater models were measured using a tensile tester with microscissors. Two different measurements were tested: tensile and microscissors tests.

Tensile tests were conducted first. Each dura-mater model or porcine dura-mater was cut in a rectangular shape at the size of $50 \mathrm{~mm}$ times $10 \mathrm{~mm}$. Figure 7 represents the experimental setup. Both ends of the tested specimens in the dura-mater models or the porcine dura-mater were sandwiched and fixed by the jigs on the tensile tester. The speed of sample stretch was set at $50 \mathrm{~mm} / \mathrm{min}$ by moving the movable head of the tensile tester (EMX-1000N-FA, IMADA, Toyohashi, Japan), and the reaction force was measured by the force sensor (DPU 50N, IMADA, Toyohashi, Japan). The stress $\sigma$ and 
stretch ratio $\lambda$ were, respectively, obtained from the measured force and displacement as shown in Equations (5) and (6),

$$
\begin{aligned}
& \sigma=\frac{F}{A} \\
& \lambda=\frac{L}{l}
\end{aligned}
$$

where $L$ represents the length of sample during tensile tests, and $l$ indicates the initial length of the sample. The test was repeated five times in each sample, and the highest and lowest values of the measured tensile stress were removed from the results.

(a)

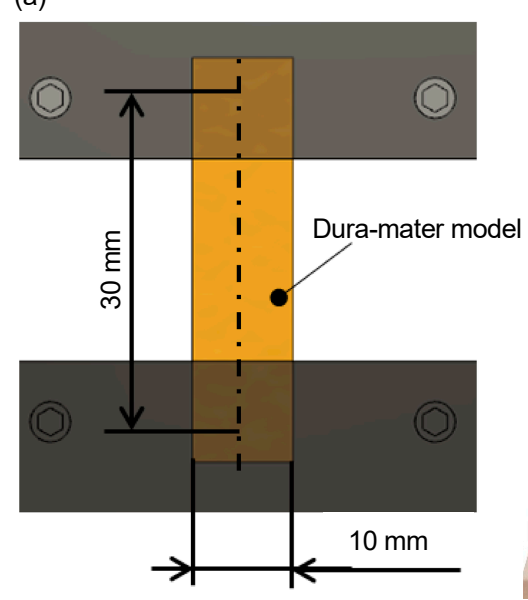

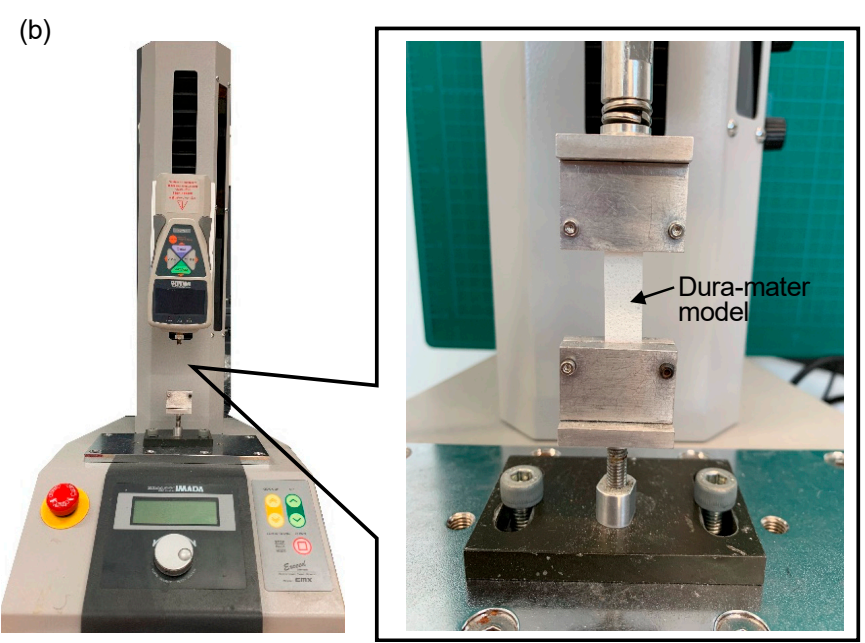

Figure 7. Tensile test: (a) Sample size for the tensile test; (b) Measurement setup for the tensile test of dura-mater models.

Figure 8 represents the relationship between $\lambda$ and $\sigma$ in each sample, and Figure 9a indicates the maximum tensile stress in each sample. The fabricated dura-mater models showed a higher stretch ratio compared with the porcine dura-mater. The maximum tensile stress of the dura-mater model in Figure 4d,e was relatively similar to that of the porcine dura-mater and there was no significant difference in statistical analysis. The tensile properties of dura-mater had a large deviation because the mechanical properties may depend on the rotation of samples. The actual dura-mater has fiber components such as collagen fibers, and those fibers have anisotropic direction in some areas [24]. Therefore, the tensile properties were changed depends on the rotation of measured samples. On the other hand, the tensile properties of fabricated dura-mater models became small because the fabricated dura-mater models were generally isotropic. As shown in Figure 9b, Young's moduli of all fabricated dura-mater models were about one-third of that of porcine dura-mater. The results of Young's moduli were caused by the elastic material used in the dura-mater models. The elastic material (acrylic rubber) was too soft (too stretchable) compared with porcine dura-mater. From the sensory test by neurosurgeons indicated (Table 3, in Section 5), the dura-mater models (b) and (e), which had relatively higher Young's moduli, showed better results. The results indicate that the dura-mater models could be modified by using a harder elastic material (a higher Young's modulus material). 


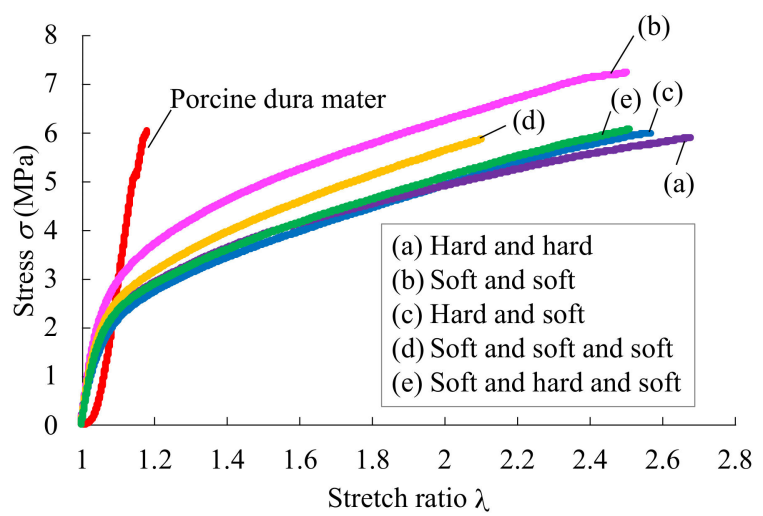

Figure 8. Experimental results of the tensile test in tested dura-matter models.

(a)

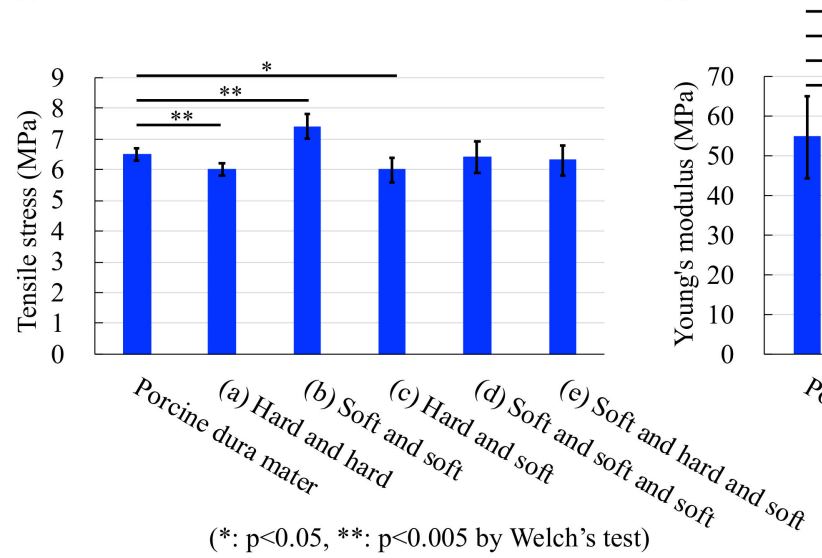

$(*: \mathrm{p}<0.05, * *: \mathrm{p}<0.005$ by Welch's test) (b)

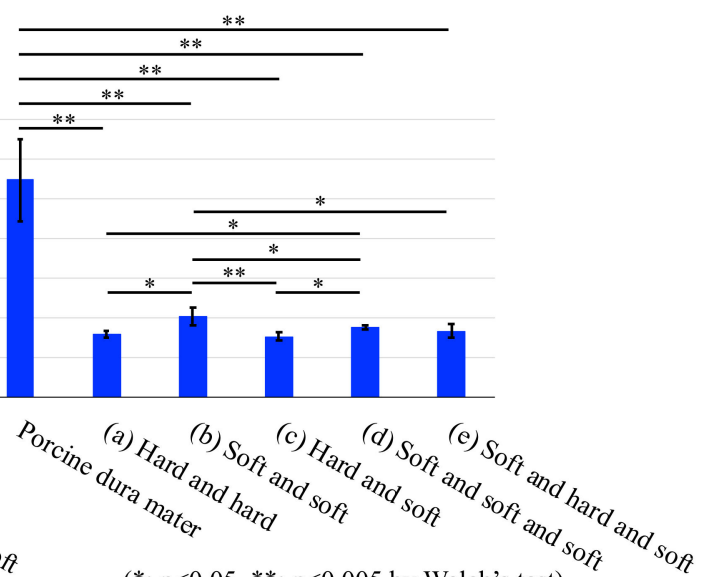

Figure 9. Experimental results of tensile measurements: (a) Tensile stress of porcine dura-mater and each dura-mater model; (b) Young's modulus of porcine dura-mater and each dura-mater model.

\subsection{Microscissors Test}

In the microscissors test, the porcine dura-mater or dura-mater models were put into a fixture, as shown in Figure 10a, and a microscissor was used to cut the dura-maters. The handle of the microscissors was pushed by the tensile tester, and the reactive forces developed during the sectioning of the porcine dura-mater or dura-mater models were measured by the force sensor. To eliminate the friction force of the microscissors itself, the reactive force when pushing the handle of microscissors without dura-mater models was also measured.

(a)

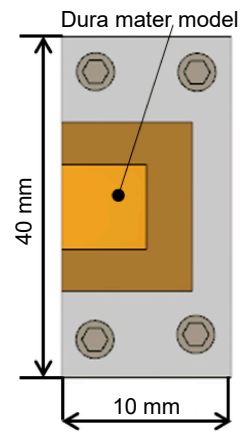

(b)

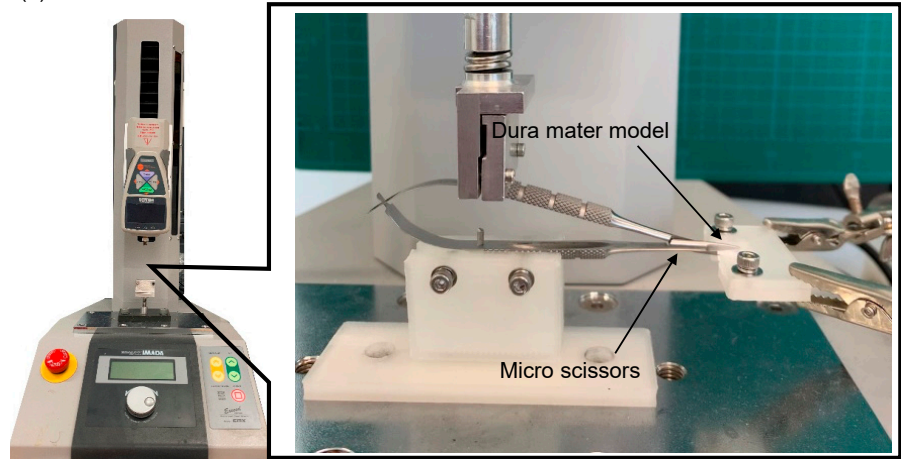

Figure 10. Experimental setup of the microscissors test in dura-matter models: (a) Design of fixture of dura-mater models; (b) Photographs of experimental setup for the microscissors test. 
Figure 11a shows the reactive force following the sectioning of the porcine dura-mater and the friction force of the microscissor itself (sectioning motion without dura-mater). The actual force required to cut the porcine dura-mater can be calculated by subtracting the frictional force from the reactive force [27]. Figure $11 \mathrm{~b}$ shows the calculated result of the difference. The same procedure was conducted for the dura-mater models shown in Figure 4a-e. Figure 12 shows the results of the microscissor tests in each dura-mater model. From the viewpoint of the maximum force as shown in Figure 13, the dura-mater model in Figure 4a (the maximum force was $0.30 \mathrm{~N}$ ) and Figure 4e (the maximum force was $0.25 \mathrm{~N}$ ) yielded relatively similar results compared with the maximum force of the porcine dura-mater of $0.27 \mathrm{~N}$. Specifically, the dura-mater model in Figure 4e yielded a similar temporal response with the cutting by microscissors and attained a maximum force during the test. However, all dura-mater models showed lower cutting force compared with porcine dura-mater with significant differences in statistical analysis as shown in Figure 13. The cutting force of dura-mater models may be increased by adding another hard non-woven fabric.

(a)

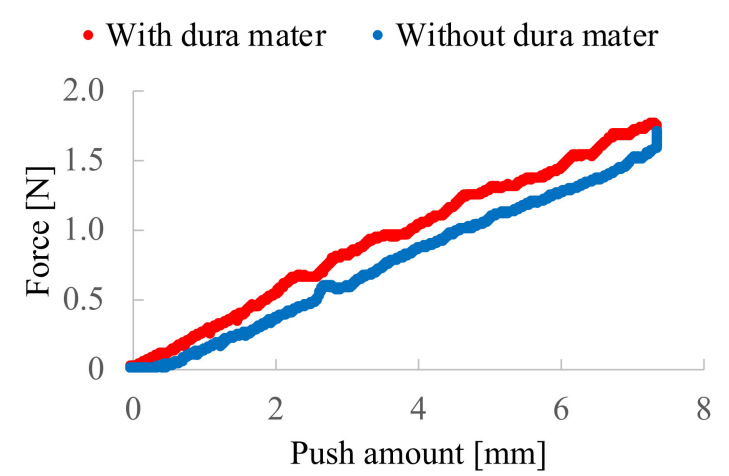

(b)

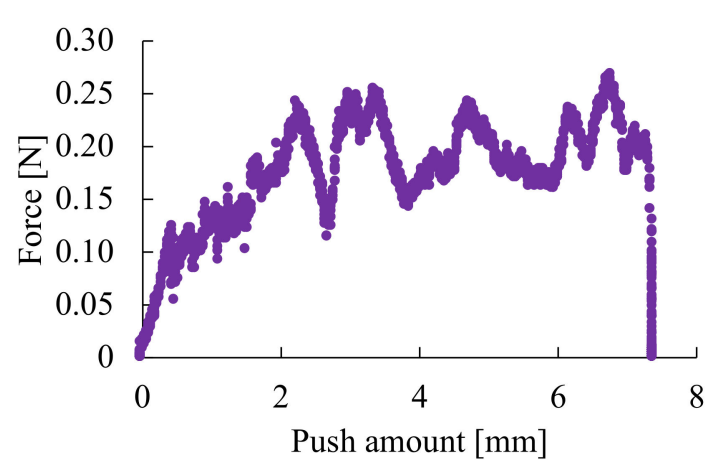

Figure 11. Results of the microscissors tests: (a) Measured force during the sectioning of the dura-mater (with dura-mater) and at the instant at which the microscissors just pushed through it (without dura-mater); (b) Cutting force difference with and without dura-mater.
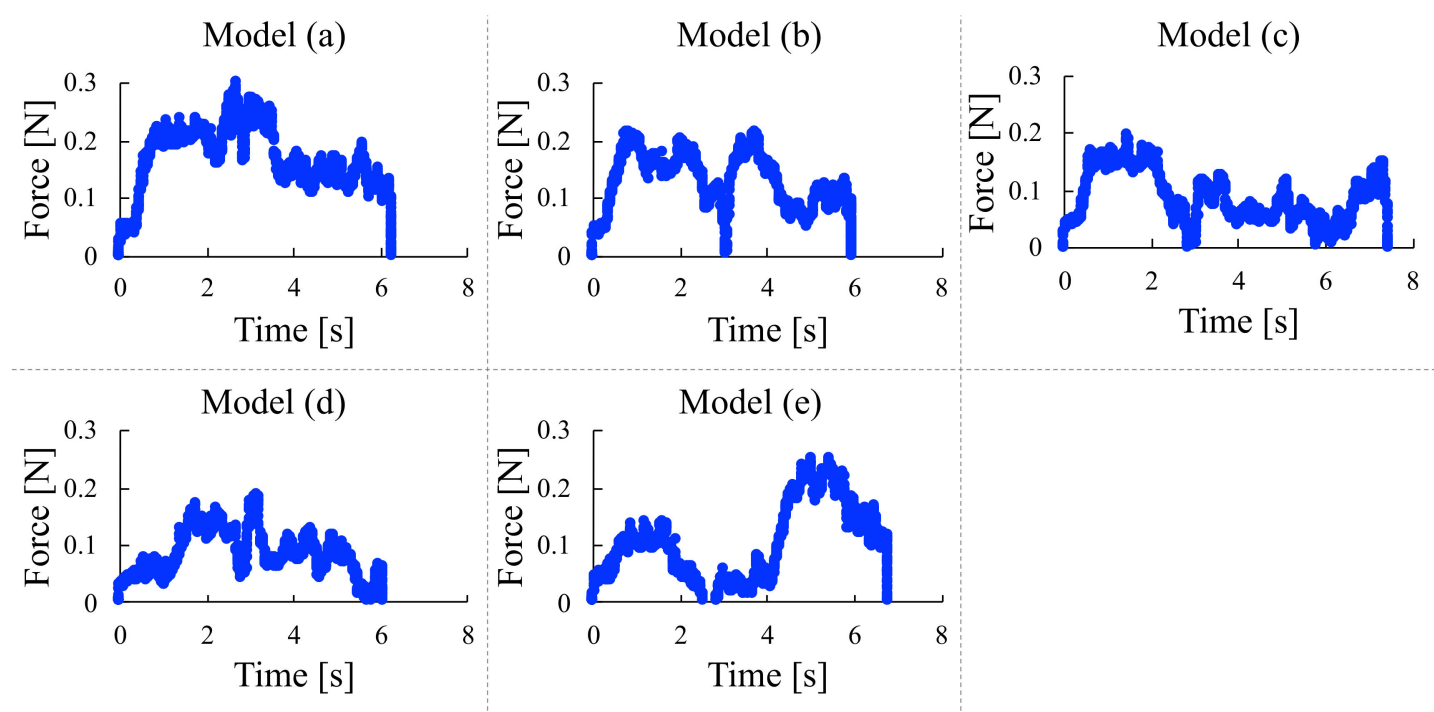

Figure 12. Experimental results of the microscissors tests in each dura-mater model (a-e). 


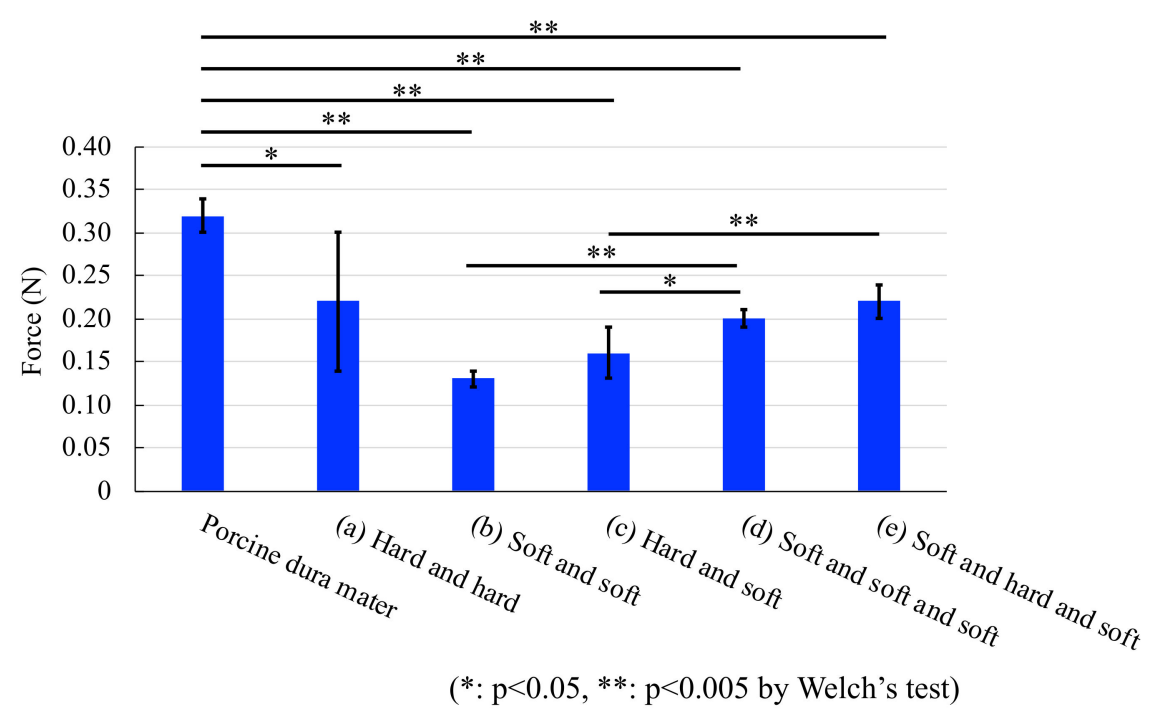

Figure 13. Experimental results of the microscissor tests.

Table 2 lists the difference between each evaluation item of the dura-mater models from the porcine dura-mater. The difference was calculated to have an error $A$ rate based on the Equation (7) listed below,

$$
A=\left|\frac{E_{\text {model }}-E_{\text {porcine }}}{E_{\text {porcine }}}\right| \times 100
$$

where $E_{\text {porcine }}$ was the measured value of porcine dura-mater, and $E_{\text {model }}$ was the measured value of the dura-mater model in each evaluation test. The results show that the dura-mater models in Figure $4 \mathrm{~d}$,e yielded similar characteristics with those generated by the porcine dura-mater regarding the tensile stress, Young's modulus, and microscissor tests, while the dura-mater model in Figure 4a yielded relatively similar characteristics with those of the porcine dura-mater regarding viscoelasticity (storage and loss moduli).

Table 2. Error rate of evaluation test (error rate [\%]).

\begin{tabular}{cccccc}
\hline & \multicolumn{5}{c}{ Dura-Mater Models } \\
\cline { 2 - 6 } - & (a) & (b) & (c) & (d) & (e) \\
\hline Storage modulus & 3.0 & 14.8 & 24.8 & 25.3 & 26.4 \\
Loss modulus & 256.2 & 635.3 & 620.4 & 531.6 & 1110 \\
Tensile stress & 9.1 & 12.4 & 8.3 & 2.4 & 4.4 \\
Young's modulus & 71.1 & 62.7 & 72.3 & 68.0 & 69.7 \\
Microscissors test & 30.1 & 58.9 & 50.8 & 38.9 & 31.7 \\
\hline
\end{tabular}

\section{Sensory Test by Neurosurgeons}

Sensory tests of fabricated dura-mater models were conducted by neurosurgeons to check whether these developed models had adequate characteristics to be used as the surgical simulator. In the tests, the dura-mater models were placed and fixed on a specimen (Figure 14a). The specimen had a rectangular hole with a size of $15 \mathrm{~mm}$ times $25 \mathrm{~mm}$, and the neurosurgeon could approach the dura-mater model through the hole by hands or surgical instruments. Two neurosurgeons tested the dura-mater models from the viewpoint of ten evaluation items: hardness on a vertical (z-direction) and a horizontal (xy-direction) direction, elasticity, surface smoothness, and brittleness, which were evaluated by touching the model by hands. Open incision with a surgical scalpel and scissors, piercing the suture needle, resistance to suture, and sewing condition were evaluated with surgical instruments. The neurosurgeons ranked the five dura-mater models from 1 (best) to 5 (worst) to show which dura-mater model was the most similar to the human dura-mater. Table 3 indicates the ranking of 
the sensory test conducted by neurosurgeons. The neurosurgeons gave high scores to the dura-mater model shown in Figure 4b,e.

In the mechanical characteristic tests, the dura-mater models shown in Figure 4d,e yielded good tensile stress, Young's modulus, and cutting force outcomes with microscissors, while the dura-mater model shown in Figure 4a yielded good viscoelasticity results. However, the neurosurgeon gave high scores to the dura-mater models shown in Figure $4 \mathrm{~b}$,e. We considered that the results were caused by two reasons. First, the fabricated dura-mater model yielded a higher viscoelastic value than that of porcine dura-mater because the porcine dura-mater had a lower viscoelastic response. Second, the dura-mater was classified as a composite material composed of cells, intercellular materials, and other interstitial substances. Consequently, Young's modulus of dura-mater could not be easily defined.

It is considered that the increase of this force and the maximum cutting force influenced the evaluation test by the neurosurgeon. The results indicated that the dura-mater model shown in Figure $4 \mathrm{e}$ that was made of acrylic rubber embedded with one hard non-woven fabric and two soft non-woven fabrics yielded similar properties as those of the human dura-mater. This model exhibited an adequate potential to be installed as a model in a surgical simulator.

(a)

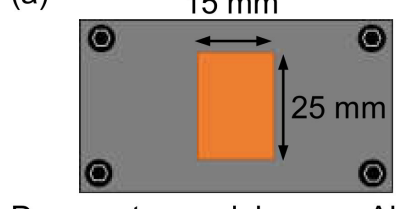

Dura-mater model

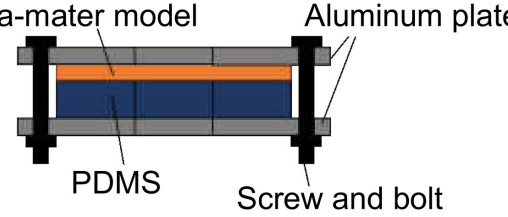

(b)

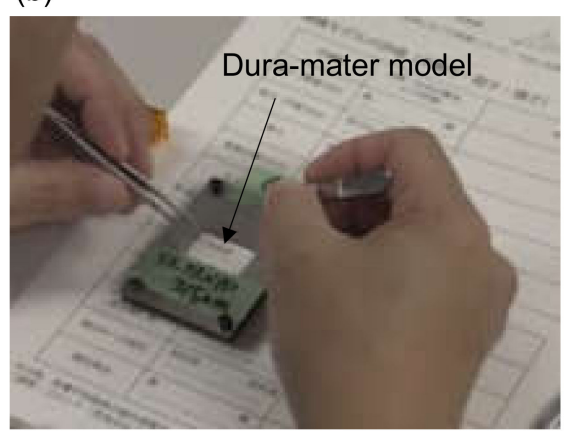

Figure 14. Sensory tests conducted by neurosurgeons: (a) Design of the specimen of the dura-mater model used for sensory tests; (b) Photograph acquired in the sensory test.

Table 3. Ranking result of sensory test by neurosurgeon (Best: 1, Worst: 5).

\begin{tabular}{cccccc}
\hline & \multicolumn{5}{c}{ Model } \\
\cline { 2 - 6 } & (a) & (b) & (c) & (d) & (e) \\
\hline Dr. M & 5 & 2 & 4 & 3 & 1 \\
Dr. K & 3 & 1 & 6 & 3 & 2 \\
\hline Total & 4 & 1 & 5 & 3 & 1 \\
\hline
\end{tabular}

\section{Integration in a Surgical Simulator}

In our project, we fabricated the surgical simulator that simulates transnasal pituitary surgery. We refer to this simulator as the "Bionic Brain" [28,29]. The fabricated dura-mater model was attached to the nasal septum model in the Bionic Brain. The blue part in Figure 15a shows the dura-mater model we fabricated. Figure $15 \mathrm{~b}$ is the nasal septum model. The dura model can be easily attached to the pituitary part of the nasal septum model. The dura-mater model is disposable, so it can be removed and replaced by a new dura-mater model in the surgical simulation. Figure $15 \mathrm{c}$ shows the integration of the dura-mater model, nasal septum model, and brain model. Figure $15 \mathrm{~d}$ is the outer appearance of the Bionic Brain after the integration of all parts. The Bionic Brain has been used as a simulation of transnasal brain surgery, as shown in Figure 16a. The fabricated dura-mater model, nasal-septum model, and all other models were checked by a neurosurgeon to ascertain whether these models had an adequate quality to be installed in surgical simulators. The neurosurgeon had access to the available surgical tools and dura-mater models through the transnasal endoscope and conducted surgical simulations with the same procedure as that used in actual transnasal surgeries, as shown 
in Figure 16b. The results indicate that the fabricated dura-mater models had an adequate quality to mimic human dura-mater in surgical simulations.
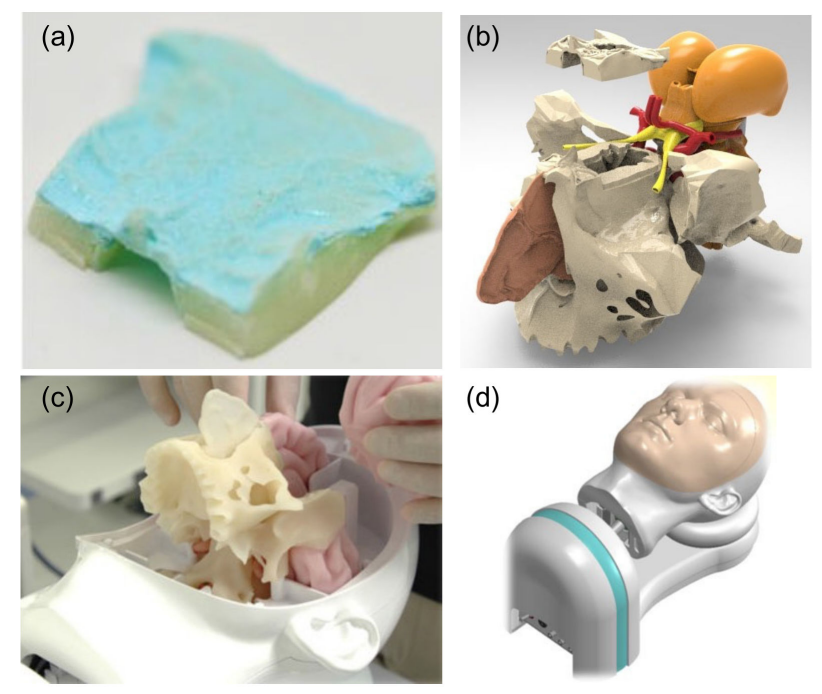

Figure 15. Dura-mater model was integrated into the Bionic Brain: (a) Dura-mater model; (b) nasal-septum model; (c) Model after integration of each model to Bionic Brain; (d) Bionic Brain for transnasal surgical simulator after covering the face model.

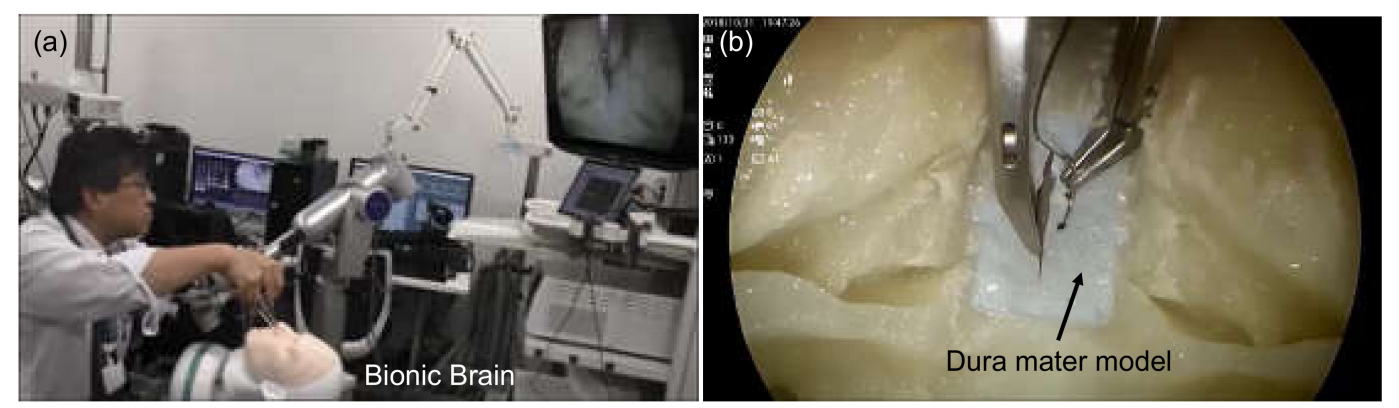

Figure 16. Surgeon sutures dura-mater model using a microscope and with other surgical tools: (a) A photograph during surgical simulation; (b) A image from the transnasal endoscope during suture of dura-mater model.

\section{Conclusions}

We developed artificial dura-mater models for a simulator of brain surgery using combinations of an elastic rubber and polypropylene fibers with two different fiber diameters. The mechanical properties, such as viscoelasticity, tensile stress, and cutting force generated by microscissors of developed dura-mater models were checked with porcine dura-mater.

Finally, developed dura-mater models were handled by neurosurgeons to ascertain whether the fabricated models exhibited adequate characteristics to be installed as a surgical simulation of brain surgery. The evaluated results indicated that the developed dura-mater made of acrylic rubber with two different non-woven fabrics had an appropriate quality to emulate human dura-mater in surgical simulations.

Author Contributions: Conceptualization, T.F. and A.H.; methodology, M.T. and A.I.; validation, M.T., A.H., and S.H.; investigation, S.H., A.H., and M.T.; resources, T.F.; data curation, M.T. and S.H.; writing-original draft preparation, M.T.; writing-review and editing, Y.H. and T.F.; visualization, M.T.; supervision, T.F.; project administration, T.F.; funding acquisition, T.F. All authors have read and agreed to the published version of the manuscript. 
Funding: This work was funded by the ImPACT Program (Bionic Humanoids Propelling New Industrial Revolution) of the Council for Science, Technology, and Innovation (Cabinet Office, Government of Japan), and JSPS Grant-in-Aid for Young Scientists (19K14946).

Conflicts of Interest: The authors declare no conflict of interest. The funders had no role in the design of the study; in the collection, analyses, or interpretation of data; in the writing of the manuscript, or in the decision to publish the results.

\section{References}

1. Fried, M.P.; Sadoughi, B.; Weghorst, S.J.; Zeltsan, M.; Cuellar, H.; Uribe, J.I.; Sasaki, C.T.; Ross, D.A.; Jacobs, J.B.; Lebowitz, R.A.; et al. Construct validity of the endoscopic sinus surgery simulator. Arch. Otolaryngol. Head Neck Surg. 2007, 133, 350-357. [CrossRef]

2. Gallagher, A.G.; Ritter, E.M.; Satava, R.M. Fundamental principles of validation, and reliability: Rigorous science for the assessment of surgical education and training. Surg. Endosc. 2003, 17, 1525-1529. [CrossRef] [PubMed]

3. Woodrum, D.T.; Andreatta, P.B.; Yellamanchilli, R.K.; Feryus, L.; Gauger, P.G.; Minter, R.M. Construct validity of the LapSim laparoscopic surgical simulator. Am. J. Surg. 2006, 191, 28-32. [CrossRef] [PubMed]

4. Maithel, S.; Sierra, R.; Korndorffer, J.; Neumann, P.; Dawson, S.; Callery, M.; Jones, D.; Scott, D. Construct and face validity of MIST-VR, Endotower, and CELTS: Are we ready for skills assessment using simulators? Surg. Endosc. 2006, 20, 104-112. [CrossRef] [PubMed]

5. Yagmurlu, K.; Safavi-Abbasi, S.; Belykh, E.; Kalani, M.Y.S.; Najahu, P.; Rhoton, A.L., Jr.; Spetzler, R.F.; Preul, M.C. Quantitative anatomical analysis and clinical experience with mini-pterional and mini-orbitozygomatic approaches for intracranial aneurysm surgery. J. Neurosurg. 2017, 127, 646-659. [CrossRef]

6. Okuda, T.; Kataoka, K.; Kato, A. Training in endoscopic endonasal transsphenoidal surgery using a skull model and eggs. Acta Neurochir. 2010, 152, 1801-1804. [CrossRef] [PubMed]

7. Wen, G.; Cong, Z.; Liu, K.; Tang, C.; Zhong, C.; Li, L.; Dai, X.; Ma, C. A practical 3D printed simulator for endoscopic endonasal transsphenoidal surgery to improve basic operational skills. Child's Nerv. Syst. 2016, 32, 1109-1116. [CrossRef]

8. Tai, B.L.; Wang, A.C.; Joseph, J.R.; Wang, P.I.; Sullivan, S.E.; McKean, E.L.; Shih, A.J.; Rooney, D.M. A physical simulator for endoscopic endonasal drilling techniques: Technical note. J. Neurosurg. 2016, 124, 811-816. [CrossRef]

9. Joseph, F.J.; Weber, S.; Raabe, A.; Bervini, D. Neurosurgical simulator for training aneurysm microsurgery-A user suitability study involving neurosurgeons and residents. Acta Neurochir. 2020, 162, 2313-2321. [CrossRef]

10. McGarvey, K.A.; Lee, J.M.; Boughner, D.R. Mechanical suitability of glycerol-preserved human dura mater for construction of prosthetic cardiac valves. Biomaterials 1984, 5, 109-117. [CrossRef]

11. Shetye, S.S.; Deault, M.M.; Puttlitz, C.M. Biaxial reaponse of ovine spinal cord dura mater. J. Mech. Behav. Biomed. Mater. 2014, 34, 146-153. [CrossRef] [PubMed]

12. Kizmazoglu, C.; Aydin, H.E.; Kaya, I.; Atar, M.; Husemoglu, B.; Kalemci, O.; Sozer, G.; Havitcioglu, H. Comparison of biomechanical properties of dura mater substitutes and cranial human dura mater: An in vitro study. J. Korean Neurosurg. Soc. 2019, 62, 635-642. [CrossRef] [PubMed]

13. Deopujari, C.E.; Karmarkar, V.S.; Shaikh, S.T.; Gadgil, U.S. Developing a dynamic simulator for endoscopic intraventricular surgeries. Child's Nerv. Syst. 2019, 35, 621-627. [CrossRef] [PubMed]

14. Ryan, J.R.; Almefty, K.K.; Nakaji, P.; Frakes, D.H. Cerebral aneurysm clipping surgery simulation using patient-specific 3D printing and silicone casting. World Neurosurg. 2016, 88, 175-181. [CrossRef] [PubMed]

15. Garling, R.J.; Jin, X.; Yang, J.; Khasawneh, A.H.; Harris, C.A. Low-cost endoscopic third ventriculostomy simulator with mimetic endoscope. J. Neurosurg. Pediatr. 2018, 22, 137-146. [CrossRef] [PubMed]

16. Craven, C.L.; Baxter, D.; Cooke, M.; Carline, L.; Alberti, S.J.M.M.; Beard, J.; Murphy, M. Development of a modelled anatomical replica for training young neurosurgeons. Br. J. Neurosurg. 2014, 28, 707-712. [CrossRef] [PubMed]

17. Eastwood, K.W.; Bodani, V.P.; Haji, F.A.; Looi, T.; Naguib, H.; Drake, J.M. Development of synthetic simulators for endoscope-assisted repair of metopic and sagittal craniosynostosis. J. Neurosurg. Pediatr. 2018, 22, 128-136. [CrossRef] 
18. Carrau, R.L.; Jho, H.D.; Ko, Y. Transnasal-transsphenoidal endoscopic surgery of the pituitary gland. Laryngoscope 1996, 106, 914-918. [CrossRef]

19. Schaberg, M.R.; Anand, V.K.; Schwartz, T.H.; Cobb, W. Microscopic versus endoscopic transnasal pituitary surgery. Curr. Opin. Otolaryngol. Head Neck Surg. 2010, 18, 8-14. [CrossRef]

20. Miller, K.; Wittek, A.; Joldes, G. Biomechanics of the brain for computer-integrated surgery. Acta Bioeng. Biomech. 2010, 12, 25-37.

21. Patin, D.J.; Eckstein, E.C.; Harum, K.; Pallares, V.S. Anatomic and biomechanical properties of human lumbar dura mater. Anesth. Analg. 1993, 76, 535-540. [CrossRef] [PubMed]

22. Reina, M.A.; Dittmann, M.; López Garcia, A.; van Zundert, A. New perspectives in the microscopic structure of human dura mater in the dorsolumbar region. Reg. Anesth. Pain Med. 1997, 22, 161-166. [PubMed]

23. Hasegawa, A.; Ichikawa, A.; Uchida, K.; Kotani, N.; Hayakawa, S.; Takeuchi, M.; Fukuda, T. Fabrication of dry dura mater models for medical surgical simulator. In Proceedings of the 2017 IEEE International Conference on Cyborg and Bionic Systems (CBS), Beijing, China, 17-19 October 2017; pp. 293-297.

24. Zwirner, J.; Scholze, M.; Waddell, J.N.; Ondruschka, B.; Hammer, N. Mechanical properties of human dura mater in tension-An analysis at an age range of 2 to 94 years. Sci. Rep. 2019, 9, 16655. [CrossRef] [PubMed]

25. Hayakawa, S.; Takeuchi, M.; Ichikawa, A.; Hasegawa, A.; Uchida, K.; Fukuda, T.; Huang, Q. Multi-layered Artificial Dura Mater Models for Medical Surgical Simulators. In Proceedings of the 2019 IEEE International Conference on Cyborg and Bionic Systems (CBS), Munich, Germany, 18-20 September 2019; pp. 310-315.

26. Yue, X.; Wang, L.; Sun, S.; Tong, L. Viscoelastic finite-element analysis of human skull-dura mater system as intracranial pressure changing. Afr. J. Biotechnol. 2008, 7, 689-695.

27. Mahvash, M.; Voo, L.M.; Kim, D.; Jeung, K.; Wainer, J.; Okamura, A.M. Modeling the forces of cutting with scissors. IEEE Trans. Biomed. Eng. 2008, 55, 848-856. [CrossRef]

28. Masuda, T.; Kanako, H.; Adachi, S.; Arai, F.; Omata, S.; Morita, A.; Kin, T.; Saito, N.; Yamashita, J.; Chinzei, K.; et al. Patients simulator for transsphenoidal surgery. In Proceedings of the 2018 International Symposium on Micro-Nano Mechatronics and Human Science (MHS), Nagoya, Japan, 9-12 December 2018; Volume 28, pp. 1-2.

29. Someya, Y.; Omata, S.; Hayakawa, T.; Mitsuishi, M.; Sugita, N.; Harada, K.; Noda, Y.; Ueta, T.; Totsuka, K.; Araki, F.; et al. Training system using Bionic-eye for internal limiting membrane peeling. In Proceedings of the 2016 International Symposium on Micro-NanoMechatronics and Human Science (MHS), Nagoya, Japan, 28-30 November 2016; pp. 67-68.

Publisher's Note: MDPI stays neutral with regard to jurisdictional claims in published maps and institutional affiliations.

(C) 2020 by the authors. Licensee MDPI, Basel, Switzerland. This article is an open access article distributed under the terms and conditions of the Creative Commons Attribution (CC BY) license (http://creativecommons.org/licenses/by/4.0/). 